\title{
Pathomechanisms of immune-mediated alopecia
}

\author{
Alessandra Anzai ${ }^{1}$, Eddy Hsi Chun Wang ${ }^{2, \oplus}$, Eunice Y. Lee ${ }^{3}$, Valeria Aoki ${ }^{1}$ and Angela M. Christiano ${ }^{2}$ \\ 1Department of Dermatology, Clinical Hospital of the University of Sao Paulo, Sao Paulo-SP 05403-900, Brazil \\ 2Department of Dermatology, Columbia University Irving Medical Center, New York, NY 10032, USA \\ ${ }^{3}$ Vagelos College of Physicians and Surgeons, Columbia University, New York, NY 10032, USA
}

Correspondence to: A. M. Christiano; E-mail: amc65@columbia.edu

Received 15 April 2019, editorial decision 22 April 2019; accepted 24 April 2019

\begin{abstract}
The hair follicle (HF) is a complex mini-organ that constantly undergoes dynamic cycles of growth and regression throughout life. While proper progression of the hair cycle requires homeostatic interplay between the HF and its immune microenvironment, specific parts of the HF, such as the bulge throughout the hair cycle and the bulb in the anagen phase, maintain relative immune privilege (IP). When this IP collapses, inflammatory infiltrates that aggregate around the bulge and bulb launch an immune attack on the HF, resulting in hair loss or alopecia. Alopecia areata (AA) and primary cicatricial alopecia (PCA) are two common forms of immune-mediated alopecias, and recent advancements in understanding their disease mechanisms have accelerated the discovery of novel treatments for immune-mediated alopecias, specifically AA. In this review, we highlight the pathomechanisms involved in both AA and CA in hopes that a deeper understanding of their underlying disease pathogenesis will encourage the development of more effective treatments that can target distinct disease pathways with greater specificity while minimizing adverse effects.
\end{abstract}

Keywords: alopecia areata, central centrifugal, frontal fibrosing, lichen planopilaris, scarring hair loss

\section{Introduction}

The word 'alopecia' originates from the Greek word 'alopex' which refers to the fox, an animal that frequently develops mange (Greek: alopecia): a skin disease caused by parasitic mites that results in fur loss. In humans, hair loss is a common clinical complaint that is a manifestation of a wide range of health conditions, including cancer, autoimmune disease and even psychiatric disorders. Hair loss often has significant impact on an individual's quality of life, as hair serves important functions in terms of one's appearance, self-esteem and sociocultural identity (1). Depression, anxiety and sleep problems are more prevalent in alopecic individuals compared with the general population (2) and, in one study, the fear of losing hair was comparable to the fear of suffering a myocardial infarction (3).

Alopecia areata $(A A)$ is a common form of immune-mediated alopecia in which an autoimmune attack on the proximal hair follicle (HF) results in non-scarring hair loss ranging in presentation from circular patches on the scalp to total scalp or full-body hair loss. Like other forms of alopecia, AA is associated with low scores on measures of quality of life in both adult and pediatric patients, as well as their families $(4,5)$. Recent advances in our understanding of AA pathogenesis, specifically with respect to the role of JAK-STAT (Janus kinase-signal transducer and activator of transcription) signaling in disease onset and progression, have been pivotal to the successful development of small-molecule JAK inhibitors (JAKi) as treatment for AA. Furthermore, the success achieved with translational $A A$ research is being adapted to develop treatments for other inflammatory hair loss disorders such as cicatricial alopecia (CA), which results in scarring hair loss.

In this review, we highlight the important discoveries made in the field of immune-mediated alopecias, as well as their clinical and histological presentations, current modes of treatment and ongoing clinical trials. We also describe the role of specific components of the HF and the hair cycle that may be involved in the onset of inflammatory hair loss. Finally, we define unanswered questions with regards to the pathogenesis of both scarring and non-scarring immune-mediated alopecias in hopes of spurring discussion that will encourage further developments in our understanding of these conditions.

The HF-a dynamic mini-organ with its own immune system

\section{Understanding the hair cycle}

The HF undergoes lifelong cycling characterized by periods of growth (anagen), involution (catagen) and rest (telogen), after which the hair is shed (exogen) and a new cycle begins 
(Fig. 1). During anagen, rapidly proliferating matrix cells, also known as transit-amplifying cells, differentiate as they move upward from the hair bulb to form one of the six layers of the HF and the hair shaft (visible part of the hair, Fig. 2). The final length of the hair shaft is determined by the rate and duration of matrix cell proliferation, which varies with the type and location of hair. The duration of anagen also varies by species; whereas the majority of human scalp HFs at any given time are in anagen, anagen in rodents, for example, lasts only a matter of days.

As the supply of proliferating matrix cells declines, the HF enters catagen, during which the lower HF regresses and hair shaft elongation ceases. This regression is driven by apoptosis of the lower HF, such that it forms an epithelial strand called the hair club that moves upward until it reaches the upper, non-cycling portion of the HF, bringing the dermal papilla in close proximity to the HF bulge (Fig. 2). This phase lasts $\sim 3$ weeks in the human scalp.

Following catagen, the HF enters a period of relative quiescence called telogen, although a wealth of recent evidence has suggested that telogen itself is also a dynamic phase of the hair cycle that is critical for maintaining HF homeostasis in preparation for the next anagen, or in response to trauma. Upon the transition to the next anagen, the club hair previously formed during catagen can be shed in a process termed exogen. Each phase of the hair cycle is governed by complex molecular mechanisms that bring together a number of signaling cascades employed by not only components of the HF itself, but also immune cells and dermal fibroblasts, as well as skin-associated adipocytes. These regulators of the hair cycle 'clock' must coordinate cell migration, differentiation, proliferation and death in order for the HF to undergo repeated cycles of regeneration and regression. As such, understanding the mechanisms that govern and modulate hair cycling has the potential to shed new light on not only skin and hair biology, but also developmental and stem cell biology, regenerative medicine and oncogenesis $(6,7)$.

\section{Anatomy of the HF-the main components}

The HF lies in close association with a sebaceous gland and arrector pili muscles, which together make up a pilosebaceous unit. The mature HF, which begins at the surface of the epidermis, can be roughly divided into a non-cycling upper portion and a lower portion that regenerates with each hair cycle.

The upper HF consists of the infundibulum, which extends from the most distal part of the HF to the opening of the sebaceous duct, as well as the isthmus, which lies between the sebaceous duct and the bulge (Fig. 2). The bulge is a critical component of maintaining the regenerative capacity of the HF, as it contains a reservoir of HF stem cells (HFSCs) from which transient amplifying cells during anagen are derived. Meanwhile, the hair bulb forms the regenerative lower $\mathrm{HF}$, and is composed of the HF matrix surrounding the mesenchymal dermal papilla. It is thought that HF induction and regeneration depend on carefully orchestrated cross-talk between the dermal papilla and the overlying epidermal matrix; furthermore, melanocytes are found among matrix cells and produce hair pigment.

Finally, in the suprabulbar region between the isthmus and the hair bulb, one can also appreciate the numerous concentric layers that form the HF epithelium, beginning with the

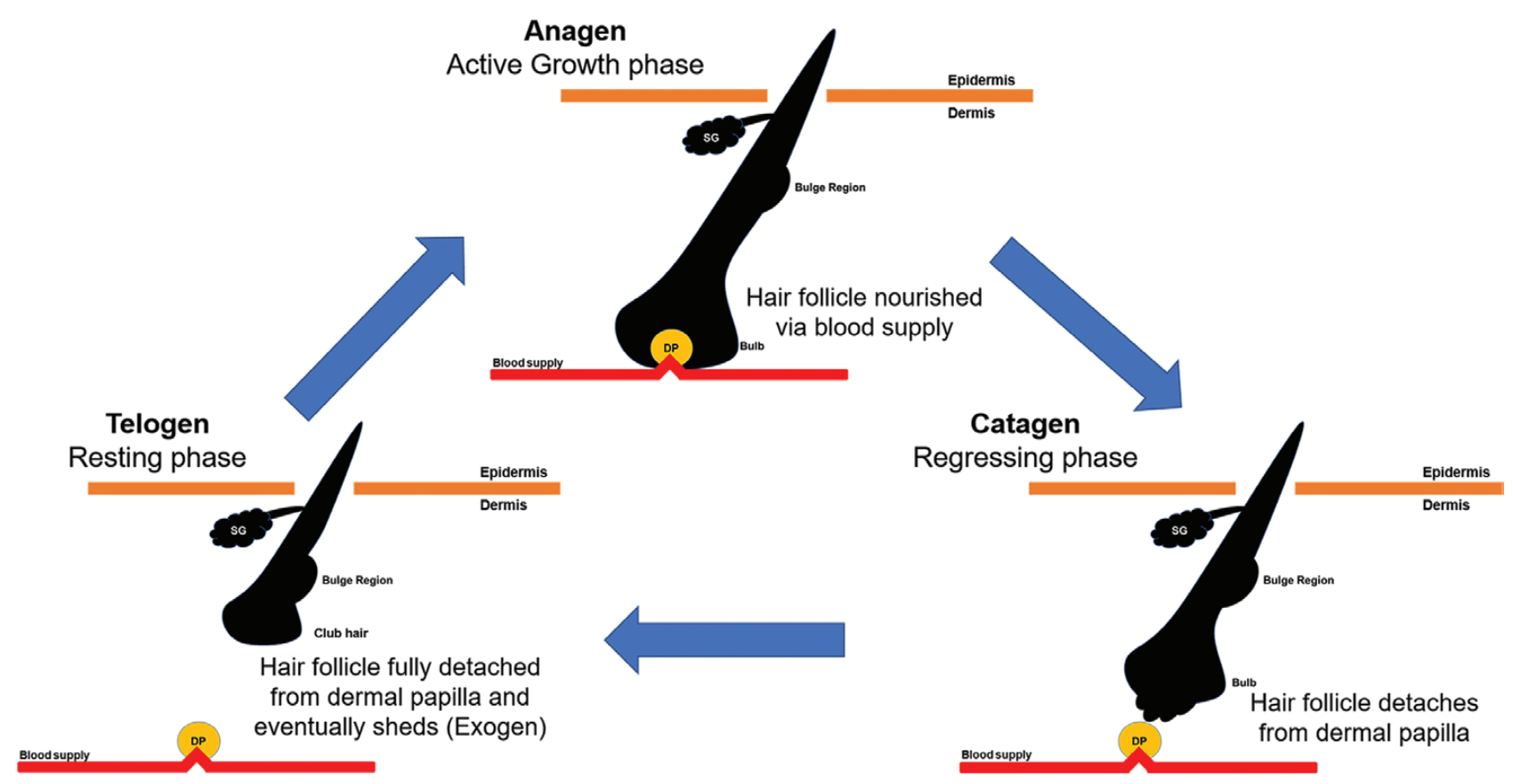

Fig. 1. Illustration of the hair growth cycle. During anagen (active growth phase), the HF is nourished via a blood vessel which supports hair growth. During catagen (regressing phase), the HF starts to regress via apoptosis and detaches from the dermal papilla and blood vessel. During telogen (resting phase), the HF rests and prepares for the next cycle. Toward the end of telogen, the hair shaft will shed (exogen) as a new hair shaft starts to grow. DP: dermal papilla; SG: sebaceous gland. 


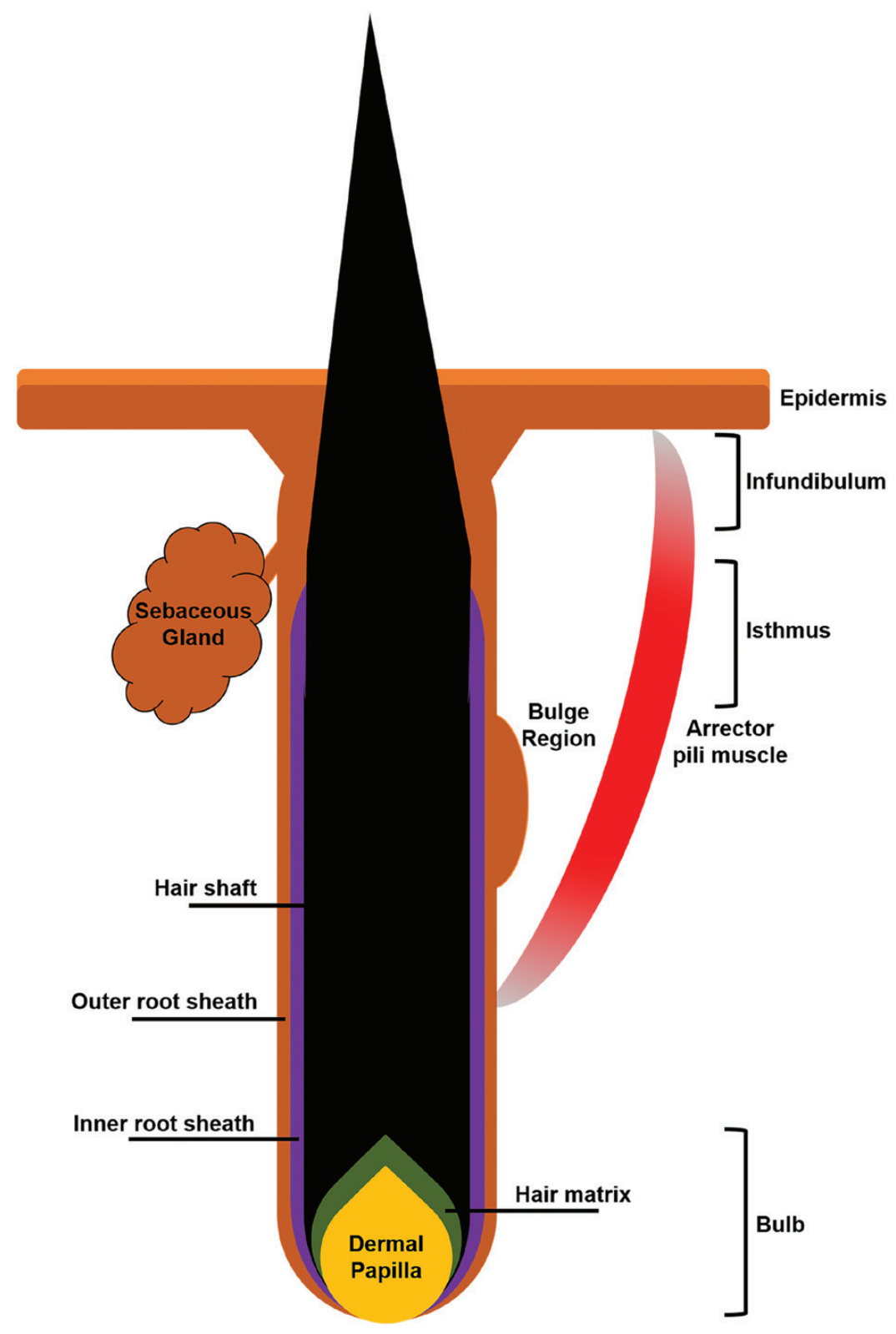

Fig. 2. Illustration of different components of a HF in anagen.

outermost outer root sheath (ORS), the inner root sheath (IRS) and finally the hair shaft, each with its own sublayers that contribute to its function in the HF.

\section{Immune privilege}

Similar to the anterior chamber of the eye, testes, brain, adrenal cortex and the fetomaternal placental unit, the HF is a site of relative immune privilege (IP), a term that was initially used to describe an environment that can tolerate allografts without rejection by the host immune system. HF IP is characterized by a number of features including the down-regulated expression of major histocompatibility complex (MHC) class I and II, an increased production of local immunosuppressants such as transforming growth factor $\beta 2$ (TGF- $\beta 2$ ), $\alpha$-melanocyte-stimulating hormone ( $\alpha-M S H)$, insulin-like growth factor 1 (IGF-1), indoleamine-pyrrole 2,3-dioxygenase (IDO) and cortisol, impaired antigen-presenting cells and an extracellular matrix barrier that prohibits infiltration of the HF by immune cells (8-10). Although HF IP was initially thought to be restricted to the anagen bulb, studies have shown that HF IP also extends to the bulge to protect the reservoir of HFSCs required for regeneration. In fact, the bulge demonstrates markedly increased expression of CD200, a type 1 transmembrane glycoprotein that interacts with the CD200 receptor to generate anti-inflammatory and tolerance signals $(11,12)$.

Thus, it has been suggested that immune-mediated alopecias such as AA and primary CA (PCA) result from an insult to HF IP, which causes inflammatory agents to infiltrate 
and attack the HF. Specifically, in AA, studies have put forth that $A A$ is an autoimmune disease mediated by $C D 8^{+} T$ cells that recognize autoantigens upon exposure to ectopic $\mathrm{MHC}$ I expression or an anagen HF bulb that has lost its IP. Similarly, in PCA, studies have demonstrated a loss of IP in the bulge region, which may be responsible for the scarring hair loss seen in PCA patients as opposed to those with AA. What triggers this loss of IP remains unknown, although possible triggers include skin microtrauma, bacterial superantigens, infectious or psychoemotional stressors, mast cell degranulation and a perifollicular/intrafollicular rise in ectopic interferon $\gamma($ IFN- $\gamma)$ secretion (12).

\section{AA-a non-scarring form of alopecia}

AA is a non-scarring alopecia that is mediated by inflammatory mechanisms and has a prevalence of $\sim 1.7-2.1 \%$ worldwide. It affects both sexes, with initial onset often occurring before 30 years of age. AA typically begins as a small, welldemarcated patch of hair loss on the scalp or beard that may spontaneously resolve without treatment. In about $30 \%$ of cases, the disease relapses; in some cases, hair loss may progress and affects the entire scalp (alopecia totalis) or the entire body (alopecia universalis) (7).

The advancement of genetic and pre-clinical studies in AA has uncovered the mechanisms involved in the pathogenesis that supported the use of small molecules for blocking specific pathways. The recent effective use of JAKi in treatment of $A A$ is an example of success for translational medicine (13). By understanding the pathomechanisms underlying other alopecias, it will be possible not only to make a more accurate diagnosis of different types of alopecia but also to develop new, effective therapies.

\section{Histology}

In acute disease, AA is histologically characterized by a 'swarm of bees' infiltration of lymphocytes, mainly composed of $\mathrm{CD}^{+}$and $\mathrm{CD}^{+} \mathrm{T}$ cells, into the peribulbar space around anagen HFs (denoted by red and yellow cells in Fig. 3, left). This intense peribulbar infiltration, which at times also invades the intrafollicular components, affects the proliferation of matrix cells and causes dystrophic shafts. AA HFs also

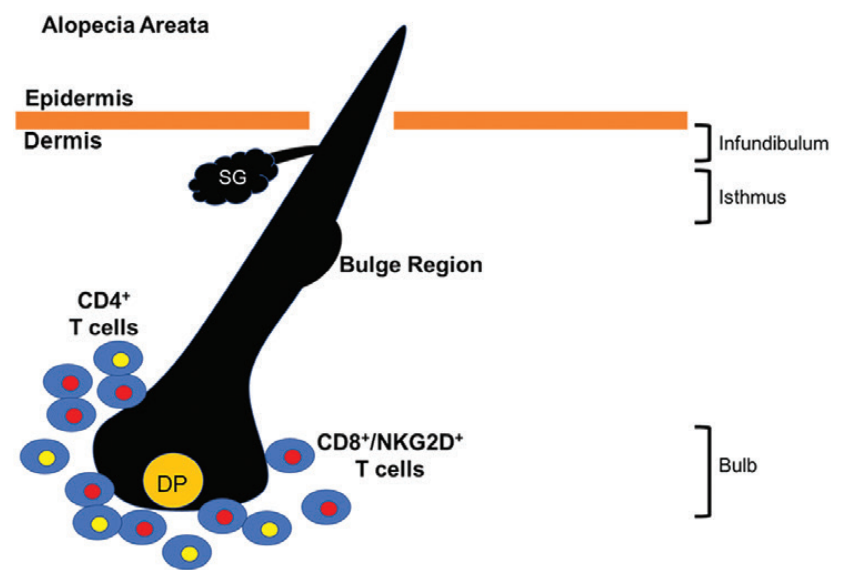

prematurely enter telogen, resulting in increased hair shedding $(10,14,15)$. Furthermore, in the chronic stages of $A A$, HFs typically become miniaturized despite a decrease in the number of inflammatory cells surrounding the peribulbar space (15). Mast cells and eosinophils can also be found in lesional AA skin throughout all stages of the disease, but their exact significance remains unknown $(9,16,17)$.

\section{Animal models}

Animal models facilitate studies of the pathogenesis and evaluation of potential new treatments, as they are in a controlled environment and are readily susceptible to genetic and cellular manipulations. However, each animal model is also associated with certain limitations and needs to be selected carefully depending on what research questions are being addressed.

The most frequently used animal models for $A A$ research are the Dundee Experimental Bald Rat (DEBR) (18), humanized severe combined immunodeficiency mice (19) and the $\mathrm{C} 3 \mathrm{H} / \mathrm{HeJ}$ AA mouse strain (20). Because of its accurate recapitulation of human $A A$ and its relative availability compared with other models, the $\mathrm{C} 3 \mathrm{H} / \mathrm{HeJ}$ AA mouse strain is currently the most well-defined and frequently used model of AA. Although it can develop AA-like hair loss spontaneously - albeit unpredictably — in old age (21), it can be induced to develop AA at a higher rate via a full-thickness skin graft from an affected donor mouse $(22,23)$. It has also been found that AA can be induced by transferring cultured cells isolated from skin-draining lymph nodes in affected mice, providing evidence that AA is a cell-mediated disease (24).

\section{Conventional treatments}

To date, there are no Food and Drugs Administration (FDA)approved treatments for $A A$ and there are few rigorous randomized controlled studies that have been published (25). The majority of currently available conventional treatments are non-specific broad immunosuppressants administered systemically or locally. First-line therapies include topical and intralesional corticosteroids, which are associated with numerous side-effects such as skin atrophy, telangiectasias and striae. Systemic corticosteroids are associated with even

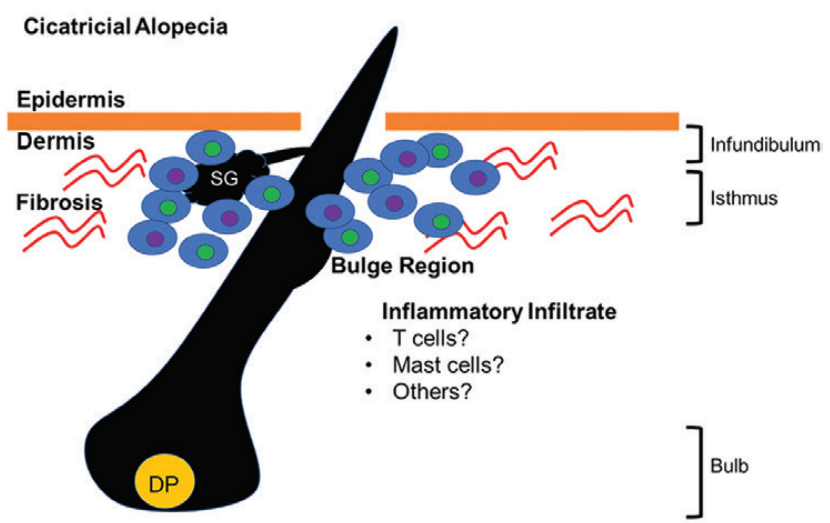

Fig. 3. Illustration of the histological presentation of AA (left) and PCA (right). DP: dermal papilla; SG: sebaceous gland. 
severer side-effects including adrenal suppression, weight gain, insomnia, glucose intolerance and acne, and thus are approved for only short-term use.

Other immunosuppressants or steroid-sparing agents such as azathioprine, methotrexate and cyclosporine also suffer from a lack of randomized trials supporting their use and warrant close patient follow-up because of potentially life-threatening immunosuppression (26). Contact immunotherapy with diphenylcyclopropenone or squaric acid dibutyl ester has also failed to produce consistent results among AA patients. As such, the non-specific nature of conventional AA treatments and their variable efficacies demand that greater efforts be made toward developing more specific forms of treatment that can better target disease-associated pathways while minimizing adverse events.

\section{AA-from bench to bedside}

In 2010, Petukhova et al. performed the first genome-wide association study (GWAS) investigating the genetic basis of AA (27). This and a later confirmatory meta-analysis performed by Betz et al. identified a number of single-nucleotide polymorphisms (SNPs) that are significantly associated with the disease (28). The studies implicated associations with several genes that control the activation and proliferation of regulatory T cells (Tregs), CTLA4, IL2RA, IL2, IL21, EOS and several human leukocyte antigen (HLA) genes, as well as genes that were already expressed in the HF itself (PRDX5 and STX17) (27). Notably, polymorphisms within the ULBP gene, which encodes activating ligands of the natural killer (NK) cell-associated receptor NKG2D, were strongly related to $A$ A but not to other autoimmune diseases.

Further functional studies in $\mathrm{C} 3 \mathrm{H} / \mathrm{HeJ}$ mice demonstrated that the transfer of $\mathrm{CD} 8{ }^{+} \mathrm{NKG} 2 \mathrm{D}^{+} \mathrm{T}$ cells from mice with $\mathrm{AA}$ induced disease in healthy mice, whereas transfer of lymphnode cell populations from diseased mice that were depleted of $\mathrm{NKG}_{2} \mathrm{D}^{+}$cells did not. It has been proposed that $\mathrm{AA}$ is mediated by $\mathrm{CD}^{+}{ }^{+} \mathrm{NKG} 2 \mathrm{D}^{+} \mathrm{T}$ cells via a positive feedback loop that involves interleukin-15 (IL-15) signaling in concert with follicular epithelial cells. Specifically, IFN- $\gamma$ produced by $\mathrm{CD}^{+}{ }^{+} \mathrm{NKG} 2 \mathrm{D}+\mathrm{T}$ cells incites the collapse of HF IP, which in turn stimulates follicular epithelial cells to produce IL-15. IL-15 is a well-established driver of cytotoxic activity by both IFN- $\gamma$-producing CD8 ${ }^{+}$cells and NK cells, and thus its production can perpetuate type I cellular autoimmunity in a feed-forward loop.

IL-15, as well as a number of other pro-inflammatory cytokines, is known to activate downstream signaling via the JAK-STAT pathway, which suggests a role for this pathway in disease pathogenesis. In fact, systemic administration of JAKi in $\mathrm{C} 3 \mathrm{H} / \mathrm{HeJ} \mathrm{AA}$ mice was shown to eliminate the IFN signature associated with AA and, furthermore, prevent disease onset. Additionally, topical administration of JAKi was able to promote hair regrowth and reverse established disease (13).

The successful use of JAKi to treat AA in pre-clinical animal models eventually led to two pilot clinical trials that successfully utilized oral JAKi_ruxolitinib or tofacitinib - to treat patients with moderate to severe $\operatorname{AA}(29,30)$. The efficacy of these inhibitors in AA treatment has since been demonstrated by a number of studies from multiple groups, summarized in another review (31). Current investigations aim to improve the specificity of these JAKi, as well as develop topical formulations in order to minimize side-effects that could result from systemic absorption of oral JAKi.

\section{Ongoing research in AA}

\section{$A A$ is driven by the recognition of autoantigens by autoreactive T cells}

Since the establishment of AA as an autoimmune disease, there has been an ongoing search for the autoantigens that could potentially trigger autoimmunity and the breach in HF IP. Several studies have suggested HF melanocyte-derived proteins as promising candidates, supported by the fact that patients first lose pigmented hairs during disease onset $(32,33)$. Earlier studies also suggest the presence of autoantibodies against HF keratinocytes (34-36).

More recently, screening of autoantigens targeted by human AA CD8 ${ }^{+} \mathrm{T}$ cells has identified trichohyalin peptides as potential triggers of disease onset (37). Computational predictions (38) as well as a case report (39) have also suggested hepatitis B virus (HBV) peptides as potential autoantigen epitopes for AA. In a different approach, highthroughput T-cell antigen receptor (TCR) sequencing has been performed to gain unbiased insight into pathogenic T-cell populations without prior knowledge of the antigen(s) that they recognize. These studies showed that AA patients showed an increase of clonality in TCRs. This indicates that there is an increased number of $\mathrm{T}$ cells that target a common antigen epitope, which corroborates the notion that AA is an antigen-driven disease (40). Continued like-minded efforts to identify autoantigens and antigen-specific $T$ cells in AA will be a crucial step to further elucidate the pathomechanisms of AA, which will prove invaluable to the development of novel therapeutics for the disease.

\section{Environmental triggers for $A A$}

Although AA has a significant genetic component, the concordance rate for AA in monozygotic twins is around $50 \%$ (41), indicating an incomplete inheritance. While the epigenetics of AA have not been fully explored, several studies have suggested a role for regulatory microRNAs in the development of $A A$. The role of other epigenetic mechanisms, which can range from post-translational histone modifications to covalent DNA modifications to other small non-coding regulatory RNAs, is still being investigated. Interestingly, microbial dysbiosis has recently been suggested as a possible mechanism underlying AA pathogenesis.

A recent report of two AA patients who exhibited hair regrowth after a fecal matter transplant (FMT) for a Clostridium difficile infection suggested that AA onset may be due to a change in the microbiome niche in the gut (42). While the role of the microbiome in AA has yet to be fully understood, the association between intestinal microbiota and autoimmunity has been reported in other autoimmune diseases including multiple sclerosis, Crohn's disease, type 1 diabetes and autoimmune uveitis (43-45). Currently, it is thought that gut microbial dysbiosis may lead to the production of bacterial 
particles that resemble self-antigens of the HF and trigger autoimmunity via a mechanism akin to molecular mimicry.

\section{Pathogenic cells in $A A$}

CD8 ${ }^{+} T$ cells, especially those that also express NKG2D, are known to be necessary and sufficient for the development of $A A(13)$. However, there are other cell types found among the pro-inflammatory milieu surrounding the HF in lesional AA skin, the roles of which have not yet been fully elucidated. Examining the contributions of non-CD8+ T cells to AA pathogenesis could help develop novel, cell-specific treatments for AA. For instance, Tregs have not only been implicated in AA via GWAS, but also are critical regulators of HF regeneration (46); thus, restoring Treg function may be a potential avenue for future treatments. $T_{h} 17$ cells have also been found in the dermis of lesional AA skin, and Lew et al. recently identified a gene polymorphism in IL17A/IL17RA that was significantly associated with $A A$ among Korean patients, suggesting a possible role for $T_{h} 17 \mathrm{~T}$ cells in disease development $(47,48)$.

\section{PCA-a scarring form of alopecia}

Unlike AA, which is a non-scarring alopecia, CA, which can be classified as primary or secondary, is characterized by the replacement of destroyed HFs with scar tissue. In PCA, the loss of HFSCs is the primary event, whereas in secondary CA, the HF is damaged by skin scarring secondary to congenital defects, trauma, inflammation, infections or neoplasms. PCAs are further subdivided by the type of inflammation detected on histological examination into lymphocytic, neutrophilic and mixed PCA (49).

Although the etiology and pathogenesis of PCA has yet to be fully understood, it is recognized that PCA onset is likely due to an autoimmune loss of HF IP, similar to AA (50). Unlike in $A A$, however, the inflammatory infiltrates in PCA target the HF bulge as opposed to the anagen bulb. The collapse of HF IP in the bulge, which is marked by increased expression of MHC class I and II, reduced CD200 expression and increased expression of IFN-inducible cytokines such as CXCL9/CXCL10/CXCL11, leads to irreversible damage to HFSCs and subsequent permanent hair loss $(51,52)$.

Lichen planopilaris (LPP) is a form of PCA that is characterized by T-cell-mediated aggression to the HF, with follicular inflammatory infiltrates in the infundibulum and isthmus near the HF bulge (53). Like AA, LPP initially manifests with localized areas of hair loss on the scalp. As the active phase progresses, however, LPP patients present with signs of perifollicular erythema and follicular hyperkeratosis in lesional skin and thus complain of itching, pain and burning (54). These signs can vanish in end-stage disease, when the discernible signs of scalp inflammation are replaced by a pattern termed 'pseudopelade of Brocq', which describes discrete, smooth, flesh-toned areas of alopecia without erythema or follicular hyperkeratosis.

There are three variants of LPP: the classical form, frontal fibrosing alopecia (FFA) and Graham-Little syndrome. The histology is the same in all subtypes (55). The classic form of LPP is usually seen between the ages of 40 and 60 , and is more common in women. Although it can affect any region of the scalp, it most often presents as localized areas of alopecia in the parietal region or vertex. Plaques can increase in size and number, converging on larger lesions and even compromising the entire scalp. The involvement of the eyelashes and hairs of the body is not common (54). Meanwhile, FFA affects the frontal hairline and often the eyebrows of postmenopausal women (56). Since the first description of FFA in 1994, increasingly more cases of FFA have been published, to the point that is considered an emerging epidemic by some authors (57). Finally, Graham-Little syndrome is a rare condition that presents with LPP lesions in the scalp alongside non-scarring alopecia of the body, as well as disseminated keratosis-pilaris lesions (55).

\section{Histology}

In all types of LPP, HFs are selectively destroyed by a chronic lymphocytic inflammatory process that spares the interfollicular region. LPP is also characterized by concentric perifollicular fibrosis, the loss of sebaceous glands, and the replacement of the follicles by scarring fibrous tracts in late-stage disease $(53,58,59)$. In addition to the CD4+ $T$ cells that infiltrate the follicular infundibulum and isthmus, a large number of mast cells can also be detected in active LPP lesions (Fig. 3, right). These mast cells are known to express the IL-23 receptor and produce IL-17A and IL-23, although their role in LPP pathogenesis has yet to be elucidated (60). There may be other cell types in the inflammatory infiltrate of LPP that have not been characterized (denoted by green and purple cells in Fig. 3, right).

\section{Conventional treatments}

First-line therapy for scarring alopecias consists of topical and/or intralesional corticosteroids. Options for systemic therapy include a short course of glucocorticoids and hydroxychloroquine, doxycycline and methotrexate (61). Pioglitazone, a thiazolidinedione and peroxisome proliferatoractivated receptor $\gamma$ (PPAR- $\gamma$ ) agonist, was used to treat LPP in a small of number of cases with mixed results (62-65). As for FFA, oral 5- $\alpha$-reductase was found to provide disease stability and reduce the rate of progression in some cases (66). More recently, due to the success of JAKi in the treatment of $A A$, a trial was performed to test the effectiveness of JAKi to treat LPP and FFA, in which 8 of 10 patients with recalcitrant disease showed clinical improvement upon administration of oral tofacitinib as either monotherapy or adjuvant therapy (67). Nonetheless, there has yet to be an established, definitive form of treatment that specifically targets the various forms of scarring alopecia, owing to the relative obscurity of their underlying disease mechanisms.

\section{Possible pathomechanisms for PCAs}

\section{Genetic components of PCAs}

A recent GWAS demonstrated a significant association with FFA at four genomic loci: 2p22.2, 6p21.1, 8q24.22 and 15q2.1. Fine-mapping within the 2p22.2 and 6p21.1 loci revealed associations with a presumed causal missense variant in CYP1B1, which encodes a member of the cytochrome P450 family, and the $H L A-B^{\star}$ 07:02 allele, respectively, suggesting 
that FFA is a genetically predisposed disorder in inflammatory and immune responses (68). A recent study also found a variant of $P A D / 3$ in central centrifugal CA (CCCA) via wholeexome sequencing. This mutation in the PAD/3 gene may result in improper formation of hair shaft, leading to the CCCA phenotype (69). Gene expression profiling demonstrated decreased tissue expression of PPAR- $\gamma$ in LPP lesions in comparison with unaffected skin, putting forth the notion that LPP might be characterized by dysregulated fatty acid and/or glucose metabolism $(70,71)$. This is further supported by the finding that mammalian target of rapamycin (mTOR) signaling pathway proteins, which are central regulators of cell metabolism, exhibit altered expression in the HFs of LPP and FFA patients compared with those of healthy controls (72).

\section{Potential external and intrinsic factors associated with PCA pathogenesis}

Potential triggers for LPP that have been previously described include scalp trauma due to head injury or hair transplant surgery (73-75), drugs, viruses and contact sensitizers $(55,76$, 77). Other studies have found an association between FFA and the use of leave-on facial skin care products and sunscreens (78-81).

Interestingly, recent evidence has suggested a role for androgens in the development of FFA: the majority of FFA patients are postmenopausal women and the disease has been associated with not only early menopause but also a high rate of concomitant androgenetic alopecia (56).

\section{Conclusion}

Identifying the pathomechanisms associated with a disease is the first step to develop treatment modalities that are highly specific and can minimize side-effects. In the field of AA, the success in rational repurposing of JAKi to reverse hair loss is a result from deciphering the pathways involved in AA pathogenesis. While many clinical trials are still ongoing to optimize this treatment for the patients, the road leading up to this point serves as a great example for the field of PCAs. Recent genetic studies and immunophenotyping of different forms of PCAs have already revealed novel targets that have clinical significance such diagnosing disease subtypes and identifying druggable pathways.

\section{Funding}

This work is supported by NIAMS Alopecia Areata Center for Research Translation (AACORT; P50AR070588). E.Y.L. is supported by the Medical Scientist Training Program at Columbia University Irving Medical Center (T32GM007367).

Conflicts of interest statement: A.M.C. is a recipient of the research grant from Pfizer and Sanofi. She is also a consultant for Aclaris Therapeutics and Dermira. A.M.C. is a shareholder of Aclaris Therapeutics. All other authors have no conflict of interest to disclose.

\section{References}

1 Marks, D. H., Penzi, L. R., Ibler, E. et al. 2019. The medical and psychosocial associations of alopecia: recognizing hair loss as more than a cosmetic concern. Am. J. Clin. Dermatol. 20:195.
2 Ghanizadeh, A. and Ayoobzadehshirazi, A. 2014. A review of psychiatric disorders comorbidities in patients with alopecia areata. Int. J. Trichology 6:2.

3 Sharma, K. H. and Jindal, A. 2014. Association between androgenetic alopecia and coronary artery disease in young male patients. Int. J. Trichology 6:5.

4 Liu, L. Y., King, B. A. and Craiglow, B. G. 2018. Alopecia areata is associated with impaired health-related quality of life: a survey of affected adults and children and their families. J. Am. Acad. Dermatol. 79:556.

5 Putterman, E., Patel, D. P., Andrade, G. et al. 2019. Severity of disease and quality of life in parents of children with alopecia areata, totalis, and universalis: a prospective, cross-sectional study. J. Am. Acad. Dermatol. 80:1389.

6 Alonso, L. and Fuchs, E. 2006. The hair cycle. J. Cell Sci. 119(Pt 3):391.

7 Breitkopf, T., Leung, G., Yu, M., Wang, E. and McElwee, K. J. 2013. The basic science of hair biology: what are the causal mechanisms for the disordered hair follicle? Dermatol. Clin. 31:1.

8 Alkhalifah, A., Alsantali, A., Wang, E., McElwee, K. J. and Shapiro, J. 2010. Alopecia areata update: part I. Clinical picture, histopathology, and pathogenesis. J Am Acad Dermatol. 62:177.

9 El Darouti, M., Marzouk, S. A. and Sharawi, E. 2000. Eosinophils in fibrous tracts and near hair bulbs: a helpful diagnostic feature of alopecia areata. J. Am. Acad. Dermatol. 42(2 Pt 1):305.

10 Strazzulla, L. C., Wang, E. H. C., Avila, L. et al. 2018. Alopecia areata: disease characteristics, clinical evaluation, and new perspectives on pathogenesis. J. Am. Acad. Dermatol. 78:1.

11 Paus, R., Bulfone-Paus, S. and Bertolini, M. 2018. Hair follicle immune privilege revisited: the key to alopecia areata management. J. Investig. Dermatol. Symp. Proc. 19:S12.

12 Paus, R., Nickoloff, B. J. and Ito, T. 2005. A 'hairy' privilege. Trends Immunol. 26:32.

13 Xing, L., Dai, Z., Jabbari, A. et al. 2014. Alopecia areata is driven by cytotoxic T lymphocytes and is reversed by JAK inhibition. Nat. Med. 20:1043.

14 Kutner, A. and Friedman, A. 2013. Hair loss in the dermatology office: an update on alopecia areata. J. Drugs Dermatol. 12:588.

15 Whiting, D. A. 2003. Histopathologic features of alopecia areata: a new look. Arch. Dermatol. 139:1555.

16 Elston, D. M., McCollough, M. L., Bergfeld, W. F., Liranzo, M. O. and Heibel, M. 1997. Eosinophils in fibrous tracts and near hair bulbs: a helpful diagnostic feature of alopecia areata. J. Am. Acad. Dermatol. 37:101.

17 Späth, U. and Steigleder, G. K. 1970. [Number of mast cells (MC) in alopecia areata]. Z. Haut. Geschlechtskr. 45:435.

18 Michie, H. J., Jahoda, C. A., Oliver, R. F. and Johnson, B. E. 1991 The DEBR rat: an animal model of human alopecia areata. Br. J. Dermatol. 125:94.

19 Gilhar, A., Keren, A., Shemer, A., d'Ovidio, R., Ullmann, Y. and Paus, R. 2013. Autoimmune disease induction in a healthy human organ: a humanized mouse model of alopecia areata. J. Invest. Dermatol. 133:844.

20 Sun, J., Silva, K. A., McElwee, K. J., King, L. E., Jr and Sundberg, J. P. 2008. The $\mathrm{C} 3 \mathrm{H} / \mathrm{HeJ}$ mouse and DEBR rat models for alopecia areata: review of preclinical drug screening approaches and results. Exp. Dermatol. 17:793.

21 Sundberg, J. P., Cordy, W. R. and King, L. E., Jr. 1994. Alopecia areata in aging $\mathrm{C} 3 \mathrm{H} / \mathrm{HeJ}$ mice. J. Invest. Dermatol. 102:847.

22 McElwee, K. J., Freyschmidt-Paul, P., Sundberg, J. P. and Hoffmann, R. 2003. The pathogenesis of alopecia areata in rodent models. J. Investig. Dermatol. Symp. Proc. 8:6.

23 McElwee, K. J., Freyschmidt-Paul, P., Zöller, M. and Hoffmann, R. 2003. Alopecia areata susceptibility in rodent models. J. Investig. Dermatol. Symp. Proc. 8:182.

24 Wang, E. H. C., Khosravi-Maharlooei, M., Jalili, R. B. et al. 2015. Transfer of alopecia areata to $\mathrm{C} 3 \mathrm{H} / \mathrm{HeJ}$ mice using cultured lymph node-derived cells. J. Invest. Dermatol. 135:2530.

25 Hordinsky, M. and Donati, A. 2014. Alopecia areata: an evidencebased treatment update. Am. J. Clin. Dermatol. 15:231.

26 Cranwell, W. C., Lai, V. W., Photiou, L. et al. 2019. Treatment of alopecia areata: an Australian expert consensus statement. Australas. J. Dermatol. 60:163. 
27 Petukhova, L., Duvic, M., Hordinsky, M. et al. 2010. Genome-wide association study in alopecia areata implicates both innate and adaptive immunity. Nature 466:113.

28 Betz, R. C., Petukhova, L., Ripke, S. et al. 2015. Genome-wide meta-analysis in alopecia areata resolves HLA associations and reveals two new susceptibility loci. Nat. Commun. 6:5966.

29 Jabbari, A., Sansaricq, F., Cerise, J. et al. 2018. An open-label pilot study to evaluate the efficacy of tofacitinib in moderate to severe patch-type alopecia areata, totalis, and universalis. J. Invest. Dermatol. 138:1539.

30 Mackay-Wiggan, J., Jabbari, A., Nguyen, N. et al. 2016. Oral ruxolitinib induces hair regrowth in patients with moderate-tosevere alopecia areata. JCl Insight 1:e89790.

31 Wang, E. H. C., Sallee, B. N., Tejeda, C. I. and Christiano, A. M. 2018. JAK inhibitors for treatment of alopecia areata. J. Invest. Dermatol. 138:1911

32 Gilhar, A., Landau, M., Assy, B., Shalaginov, R., Serafimovich, S. and Kalish, R. S. 2001. Melanocyte-associated T cell epitopes can function as autoantigens for transfer of alopecia areata to human scalp explants on Prkdc(scid) mice. J. Invest. Dermatol. 117:1357.

33 Paus, R., Slominski, A. and Czarnetzki, B. M. 1993. Is alopecia areata an autoimmune-response against melanogenesis-related proteins, exposed by abnormal MHC class I expression in the anagen hair bulb? Yale J. Biol. Med. 66:541.

34 Leung, M. C., Sutton, C. W., Fenton, D. A. and Tobin, D. J. 2010. Trichohyalin is a potential major autoantigen in human alopecia areata. J. Proteome Res. 9:5153.

35 Tobin, D. J., Orentreich, N., Fenton, D. A. and Bystryn, J. C. 1994. Antibodies to hair follicles in alopecia areata. J. Invest. Dermatol. 102:721.

36 Tobin, D. J., Sundberg, J. P., King, L. E., Jr, Boggess, D. and Bystryn, J. C. 1997. Autoantibodies to hair follicles in $\mathrm{C} 3 \mathrm{H} / \mathrm{HeJ}$ mice with alopecia areata-like hair loss. J. Invest. Dermatol. 109:329.

37 Wang, E. H. C., Yu, M., Breitkopf, T. et al. 2016. Identification of autoantigen epitopes in alopecia areata. J. Invest. Dermatol. 136:1617.

38 Richardson, C. T., Hayden, M. S., Gilmore, E. S. and Poligone, B. 2018. Evaluation of the relationship between alopecia areata and viral antigen exposure. Am. J. Clin. Dermatol. 19:119.

39 Chu, C. H., Cheng, Y. P. and Chan, J. Y. 2016. Alopecia areata after vaccination: recurrence with rechallenge. Pediatr. Dermatol. 33:e218.

40 de Jong, A., Jabbari, A., Dai, Z. et al. 2018. High-throughput T cell receptor sequencing identifies clonally expanded CD8+ T cell populations in alopecia areata. JCI Insight 3:e121949.

41 Rodriguez, T. A., Fernandes, K. E., Dresser, K. L. and Duvic, M.; National Alopecia Areata Registry. 2010. Concordance rate of alopecia areata in identical twins supports both genetic and environmental factors. J. Am. Acad. Dermatol. 62:525.

42 Rebello, D., Wang, E., Yen, E., Lio, P. A. and Kelly, C. R. 2017. Hair growth in two alopecia patients after fecal microbiota transplant. ACG Case Rep. J. 4:e107.

43 Horai, R., Sen, H. N. and Caspi, R. R. 2017. Commensal microbiota as a potential trigger of autoimmune uveitis. Expert Rev. Clin. Immunol. 13:291.

44 Li, B., Selmi, C., Tang, R., Gershwin, M. E. and Ma, X. 2018. The microbiome and autoimmunity: a paradigm from the gut-liver axis. Cell. Mol. Immunol. 15:595.

45 Opazo, M. C., Ortega-Rocha, E. M., Coronado-Arrázola, I. et al. 2018. Intestinal microbiota influences non-intestinal related autoimmune diseases. Front. Microbiol. 9:432.

46 Ali, N., Zirak, B., Rodriguez, R. S. et al. 2017. Regulatory T cells in skin facilitate epithelial stem cell differentiation. Cell 169:1119.

47 Lew, B. L., Cho, H. R., Haw, S., Kim, H. J., Chung, J. H. and Sim, W. Y. 2012. Association between IL17A/IL17RA gene polymorphisms and susceptibility to alopecia areata in the Korean population. Ann. Dermatol. 24:61.

48 Tanemura, A., Oiso, N., Nakano, M., Itoi, S., Kawada, A. and Katayama, I. 2013. Alopecia areata: infiltration of Th17 cells in the dermis, particularly around hair follicles. Dermatology 226:333.
49 Olsen, E. A., Bergfeld, W. F., Cotsarelis, G. et al.; Workshop on Cicatricial Alopecia. 2003. Summary of North American Hair Research Society (NAHRS)-sponsored workshop on cicatricial alopecia, Duke University Medical Center, February 10 and 11, 2001. J. Am. Acad. Dermatol. 48:103.

50 Harries, M. J., Jimenez, F., Izeta, A. et al. 2018. Lichen planopilaris and frontal fibrosing alopecia as model epithelial stem cell diseases. Trends Mol. Med. 24:435

51 Harries, M. J., Meyer, K., Chaudhry, I. et al. 2013. Lichen planopilaris is characterized by immune privilege collapse of the hair follicle's epithelial stem cell niche. J. Pathol. 231:236.

52 Harries, M. J. and Paus, R. 2010. The pathogenesis of primary cicatricial alopecias. Am. J. Pathol. 177:2152.

53 Mehregan, D. A., Van Hale, H. M. and Muller, S. A. 1992. Lichen planopilaris: clinical and pathologic study of forty-five patients. J. Am. Acad. Dermatol. 27(6 Pt 1):935.

54 Kang, H., Alzolibani, A. A., Otberg, N. and Shapiro, J. 2008. Lichen planopilaris. Dermatol. Ther. 21:249.

55 Assouly, P. and Reygagne, P. 2009. Lichen planopilaris: update on diagnosis and treatment. Semin. Cutan. Med. Surg. 28:3.

56 Vañó-Galván, S., Molina-Ruiz, A. M., Serrano-Falcón, C. et al. 2014. Frontal fibrosing alopecia: a multicenter review of 355 patients. J. Am. Acad. Dermatol. 70:670.

57 Mirmirani, P., Tosti, A., Goldberg, L., Whiting, D. and Sotoodian, B. 2019. Frontal fibrosing alopecia: an emerging epidemic. Skin Appendage Disord. 5:90.

58 Matta, M., Kibbi, A. G., Khattar, J., Salman, S. M. and Zaynoun, S. T. 1990. Lichen planopilaris: a clinicopathologic study. J. Am. Acad. Dermatol. 22:594.

59 Tandon, Y. K., Somani, N., Cevasco, N. C. and Bergfeld, W. F. 2008. A histologic review of 27 patients with lichen planopilaris. J. Am. Acad. Dermatol. 59:91.

60 Hobo, A., Harada, K., Maeda, T. et al. 2018. IL-17-positive mast cell infiltration in the lesional skin of lichen planopilaris: possible role of mast cells in inducing inflammation and dermal fibrosis in cicatricial alopecia. Exp. Dermatol. in press, doi: 10.1111/exd.13816

61 Rácz, E., Gho, C., Moorman, P. W., Noordhoek Hegt, V. and Neumann, H. A. 2013. Treatment of frontal fibrosing alopecia and lichen planopilaris: a systematic review. J. Eur. Acad. Dermatol. Venereol. 27:1461.

62 Baibergenova, A. and Walsh, S. 2012. Use of pioglitazone in patients with lichen planopilaris. J. Cutan. Med. Surg. 16:97.

63 Mesinkovska, N. A., Tellez, A., Dawes, D., Piliang, M. and Bergfeld, W. 2015. The use of oral pioglitazone in the treatment of lichen planopilaris. J. Am. Acad. Dermatol. 72:355.

64 Mirmirani, P. and Karnik, P. 2009. Lichen planopilaris treated with a peroxisome proliferator-activated receptor gamma agonist. Arch. Dermatol. 145:1363.

65 Spring, P., Spanou, Z. and de Viragh, P. A. 2013. Lichen planopilaris treated by the peroxisome proliferator activated receptor- $\gamma$ agonist pioglitazone: lack of lasting improvement or cure in the majority of patients. J. Am. Acad. Dermatol. 69:830.

66 Murad, A. and Bergfeld, W. 2018. 5-Alpha-reductase inhibitor treatment for frontal fibrosing alopecia: an evidence-based treatment update. J. Eur. Acad. Dermatol. Venereol. in press, doi: $10.1111 / j d v .14930$

67 Yang, C. C., Khanna, T., Sallee, B., Christiano, A. M. and Bordone, L. A. 2018. Tofacitinib for the treatment of lichen planopilaris: a case series. Dermatol. Ther. 31:e12656.

68 Tziotzios, C., Petridis, C., Dand, N. et al. 2019. Genome-wide association study in frontal fibrosing alopecia identifies four susceptibility loci including HLA-B*07:02. Nat. Commun. 10:1150.

69 Malki, L., Sarig, O., Romano, M. T. et al. 2019. Variant PADI3 in central centrifugal cicatricial alopecia. N. Engl. J. Med. 380:833.

70 Harries, M. J. and Paus, R. 2009. Scarring alopecia and the PPAR-gamma connection. J. Invest. Dermatol. 129:1066.

71 Karnik, P., Tekeste, Z., McCormick, T. S. et al. 2009. Hair follicle stem cell-specific PPARgamma deletion causes scarring alopecia. J. Invest. Dermatol. 129:1243.

72 Dicle, O., Celik-Ozenci, C., Sahin, P. et al. 2018. Differential expression of mTOR signaling pathway proteins in lichen planopilaris and frontal fibrosing alopecia. Acta Histochem. 120:837. 
73 Chiang, Y. Z., Tosti, A., Chaudhry, I. H. et al. 2012. Lichen planopilaris following hair transplantation and face-lift surgery. Br. J. Dermatol. 166:666.

74 Donovan, J. 2012. Lichen planopilaris after hair transplantation: report of 17 cases. Dermatol. Surg. 38:1998.

75 Montpellier, R. A. and Donovan, J. C. 2014. Scalp trauma: a risk factor for lichen planopilaris? J. Cutan. Med. Surg. 18:214.

76 Chieregato, C., Zini, A., Barba, A., Magnanini, M. and Rosina, P. 2003. Lichen planopilaris: report of 30 cases and review of the literature. Int. J. Dermatol. 42:342.

77 Jayasekera, P. S., Walsh, M. L., Hurrell, D. and Parslew, R. A. 2016. Case report of lichen planopilaris occurring in a pediatric patient receiving a tumor necrosis factor $\alpha$ inhibitor and a review of the literature. Pediatr. Dermatol. 33:e143.
78 Aldoori, N., Dobson, K., Holden, C. R., McDonagh, A. J., Harries, M. and Messenger, A. G. 2016. Frontal fibrosing alopecia possible association with leave-on facial skin care products and sunscreens; a questionnaire study. Br. J. Dermatol. 175:762.

79 Cranwell, W. C. and Sinclair, R. 2019. Sunscreen and facial skincare products in frontal fibrosing alopecia: a case-control study. Br. J. Dermatol. 180:943.

80 Debroy Kidambi, A., Dobson, K., Holmes, S. et al. 2017. Frontal fibrosing alopecia in men: an association with facial moisturizers and sunscreens. Br. J. Dermatol. 177:260

81 Moreno-Arrones, O. M., Saceda-Corralo, D., RodriguesBarata, A. R. et al. 2019. Risk factors associated with frontal fibrosing alopecia: a multicentre case-control study. Clin. Exp. Dermatol. 44:404. 